Article

\title{
Development of a Platform for Securing Interoperability between Components in a Carbon-Free Island Microgrid Energy Management System
}

\author{
Jihui Hwang ${ }^{1}$, Yun-Sik Oh ${ }^{2, * \mathbb{D}}$, Jin-Uk Song ${ }^{2}$, Jae-Guk An ${ }^{2}$ and Jin-Hong Jeon ${ }^{1}$ \\ 1 Smart Grid Research Division Distributed Power System Research Center, \\ Korea Electrotechnology Research Institute, Gwangju 61751, Korea; jhwang@keri.re.kr (J.H.); \\ jhjeon@keri.re.kr (J.-H.J.) \\ 2 Department of Electrical Engineering, Kyungnam University, Changwon 51767, Korea; \\ sgu7484@gmail.com (J.-U.S.); dks3752@gmail.com (J.-G.A.) \\ * Correspondence: fivebal2@kyungnam.ac.kr
}

check for updates

Citation: Hwang, J.; Oh, Y.-S.; Song, J.-U.; An, J.-G.; Jeon, J.-H.

Development of a Platform for Securing Interoperability between Components in a Carbon-Free Island Microgrid Energy Management System. Energies 2021, 14, 8525. https://doi.org/10.3390/en14248525

Academic Editor:

Antonio Cano-Ortega

Received: 9 November 2021

Accepted: 13 December 2021

Published: 17 December 202

Publisher's Note: MDPI stays neutral with regard to jurisdictional claims in published maps and institutional affiliations.

Copyright: (c) 2021 by the authors. Licensee MDPI, Basel, Switzerland. This article is an open access article distributed under the terms and conditions of the Creative Commons Attribution (CC BY) license (https:// creativecommons.org/licenses/by/ $4.0 /)$

\begin{abstract}
The rigidity of information technology (IT) has been hindering the development of various businesses regarding energy management systems (EMSs) of power networks, although this area has become more diversified, resulting in changes of elements in the systems due to the introduction of renewable energy (RE) and the new energy industry. In order to effectively accommodate these changes, EMSs should be developed in a structure with a standard-based interface, which can secure interoperability between components in the EMS. In previous studies, the common information model (CIM) proposed by IEC TC57 has been utilized for developing EMSs of power networks, but there are gaps between the existing CIM and an information model for the EMSs of carbon-free island microgrids (MGs), which are a newly introduced form of power network covering multiple islands for reducing carbon emissions. This paper proposes a CIM-based software platform for a carbon-free island MG-EMS to efficiently operate the power network and secure interoperability between components in the MG-EMS. Concerning service restoration of the power network, use cases and business objects representing information exchanged between the components in the EMS are derived, and the existing CIM is extended based on the results of the gap analysis in order to provide necessary information on the MG-EMS. The validity of the proposed platform is verified by exchanging payloads between components in the MG-EMS based on the profile extracted from the extended CIM. Furthermore, the performance of the proposed platform regarding data size and speed of data exchange is presented. Based on the case study results, it is concluded that the proposed platform based on the extended CIM can exchange data between the components in the MG-EMS, achieving reasonable data size and speed of data exchange with the help of the interoperability between components in the carbon-free island MG-EMS.
\end{abstract}

Keywords: energy management system (EMS); carbon-free island; microgrid (MG); interoperability; common information model (CIM)

\section{Introduction}

According to the Renewable Energy 3020 Implementation Plan (signifying that in 2030 there should be $20 \%$ of energy from renewable sources) and the Korean government's declaration of being carbon-free by 2050, the introduction of renewable energy (RE) and energy storage systems (ESSs) in Korea has been increasing rapidly [1]. The Korean government has established various policies for hosting a large amount of RE-mainly photovoltaic (PV) and wind power generation-and the goal of supplying RE will be expanded to $14.3 \%$ of primary energy and $21.6 \%$ of total power generation by 2030 [2] In addition, a new energy industry in the electric power sector, entailing the integrated operation of power generation and demand resources, has attracted attention. With the 
trend mentioned above, projects are underway to build applications using big data by grafting information technology (IT) into the conventional energy management systems (EMSs) [3]. Various services will be introduced in the future due to the increase in RE and the expansion of the new energy industry; accordingly, future EMSs should be designed in a structure that can respond to these newly introduced services. In order to build a software platform accommodating various services, interoperability should be secured in advance.

IEC TC57 deals with technical standards on information exchange in power systems, including EMSs, supervisory control and data acquisition (SCADA), and distribution automation. IEC 61850 specifies a standard on information and communication technology (ICT) between system and legacy local equipment. In addition, IEC 61970 stipulates standards on information models regarding EMSs and SCADA, which is known as the common information model (CIM) [4]. IEC 61968 expands the common information model for distribution automation, and specifies a standard for information exchange methods of the information model included in IEC 61970.

Various studies related to the interoperability between a system and its components have been conducted. The authors of [5] proposed a method to apply interoperability through IEC 61968/61970 and IEC 61850 data converters. In [6], a method of integrating IEC 61970-301, covering the power management part, and IEC 61850 dealing with the control and protection of substations, is presented by using Query/View/Transformation (QVT). A scheme of using CIM for data modeling of the shipment power system analysis is proposed in $[7,8]$, pointing out that problems may arise when editing or merging graphics, since existing power system graphics applications use an absolute coordinate approach. For solving the problems, the expansion of the IEC 61970-453 graphic model is proposed. A method to build a metadata storage model was proposed in [9] to solve the problem of the metadata model wherein multiple IDs are merged within the storage in the CIM. A methodology for automation that requires manual mapping is suggested in [10], since there is no standard for interaction between IEC 61850-based systems and IEC 61970-based systems. The authors of [11] found that it is difficult to maintain the integrity of distribution networks concerning databases (DBs) due to changes in network topology, and proposed a solution using the CIM and CIM graphics. In [12], the need for applying the CIM to secure interoperability between applications developed in Europe is presented, and a model-based software engineering method is proposed. The authors of [13] proposed a CIM design plan to maintain the CIM-based standard. In [14], the expansion of the CIM to express the node-breaker model and bus-branch model by adding the 'TopologyBranch' class is suggested. The authors of [15] highlight the necessity of the CIM, which enables the communication between a system and its applications, in order to secure interoperability of the distribution system; in addition, an information model for distribution lines, distribution loads, and some specific distribution devices based on the CIM is proposed. The authors of [16] presented a method of utilizing the BPMN standard to overcome the problem of expressing temporal processes due to UML objects. An integrated common simulation environment for real-time evaluation of power and ICT systems is proposed in [17], using high-level architecture (HLA). The authors of [18] proposed a data-based approach for the visualization of power systems. A design of CIMGDB, storing and processing connected data in power system applications, is presented in [19]. A solution for handling the expansion of the CIM standard and maintaining the compatibility of previous versions, without sacrificing a higher level of detail, is suggested in [20]. A method for integrating IEC 61850 and 61970 based on a meta-modeling approach is presented in [21]. However, the data sharing and analysis on the operational status of each device are not possible since the types, functions, and interfaces of various devices are different during monitoring. An information model for a wide-area management system (WAMS) based on IEC 61970, and a scheme for extending the line objects in IEC 61850 and 61970 to ring circuit and circuit section objects, are proposed in [22,23]. A new graphics system according to IEC 61970, and a method to implement mediator synthesis to connect components (MICS) that maps 
interfaces for inter-component linkage, are proposed in [24,25]. A modeling approach to evaluate the high performance and real-time performance of IEC 61850-based substation automation systems is presented in [26].

Most of the studies mentioned above deal with the extension of the IEC 61970 CIM and studies on methods to ensure 1:1 and 1:N interoperability between systems and/or between components. Moreover, the extensions have been made to operate existing power networks, not newly introduced ones. In general, non-standard interfaces are made when developing the EMSs of new power networks such as the carbon-free island MG. However, it is hard to respond to changes in input/output data due to revisions of existing components or the adoption of new components under the non-standard interface environment. Therefore, the design of the CIM should satisfy the requirements for operating the carbon-free island $\mathrm{MG}$, and a platform with interoperability between the components in the EMS should be made based on the CIM.

The MG-EMS is a system used by operators of electric utility grids to monitor, control, and optimize the performance of MGs in order to ensure reliable, resilient, and economical operations. The authors of [27] proposed MG-EMS frameworks consisting of forecast, optimization, data analysis, and human-machine interface. In [28,29], the algorithms for managing energy in an MG are presented. The MG-EMS of carbon-free islands proposed in this paper, linked through DC networks, consists of power flow, voltage control, prediction of generation and load, energy demand management, protection coordination, and fault location, isolation, and restoration (FLISR).

In this paper, a platform for ensuring interoperability between the internal components of an MG-EMS for carbon-free islands based on the CIM-a common information model presented in IEC 61970 and 61968-is proposed. Many studies have been conducted on the CIM extension according to the real system, such as distribution systems, ship power systems, etc., for systems, components, and processes $[11,15,30,31]$ or the integration of different standards, such as IEC61850, IEC61970, etc. In this paper, a method was proposed for developing MG-EMS platforms applicable to reality as well as the CIM extension for carbonfree islands linked through DC networks. As the data exchange in a structure with the standard-based interface can be achieved by utilizing the proposed platform based on the extended CIM, it is possible to efficiently respond to changes in the industry and relevant services such as future energy industry strategies. Furthermore, other systems/components can be developed independently from the MG-EMS without mutual consultation, leading to reductions in the time and costs for developing new systems/components, since the proposed MG-EMS platform has a standard-based interface.

The structure of this paper is as follows: Section 2 deals with the purpose, function, and composition of the carbon-free island MG-EMS. In Section 3, the purpose, necessity, scope of utilization, and expected effect of ensuring the interoperability of the EMS are analyzed. In addition, a plan to secure the interoperability of the EMS platform is presented in Section 4. Finally, in Section 5, the usefulness of the scheme proposed in this paper for ensuring interoperability between components is verified by conducting multiple case studies on the proposed scheme.

\section{Carbon-Free Island MG-EMS}

\subsection{Overview of Carbon-Free Island MGs}

An eco-friendly carbon-free island is an island that supplies the energy consumed within the island through RE such as PV, wind power generation, geothermal heat, etc. It is possible to solve greenhouse gas emissions and relevant environmental problems caused by fuel transportation and related accidents by self-generating and operating electricity through the carbon-free island. The island was promoted to expand RE supply and solve environmental problems, but economic and technical problems have arisen due to high-density RE. As a result of the demonstration of 11 islands-including Gapa-do, Baeka-do, Samma-do, and Sangtae-do-various problems have occurred, such as reduced 
generator utilization rate, financial and electrical losses for RE, and effects on the reliability of facilities.

It is necessary to convert an island to one with carbon-free capability, since power generation for smaller islands costs more. However, it is difficult to obtain suitable RE resources that satisfy reliability and economic feasibility. In addition, due to the geographical and environmental characteristics of the island area, it is vulnerable to natural disasters such as typhoons, and the ripple effect of accidents within the island power network and failures of major facilities is very large. Each carbon-free island with high penetration of RE faces difficulties in balancing supply and demand in response to unexpected changes in climate or load consumption patterns. Significant power loss may occur due to excessive installation of RE, and facility investment in ESSs may be overly introduced.

Each island grid can be interconnected by using DC networks and electric ships, and can be operated in an integrated strategy so that the energy can be transferred between islands in order to improve the utilization rate of RE and the economic feasibility. Figure 1 shows an example system of carbon-free islands linked through DC networks. It is possible to overcome the limitations of small island power networks by utilizing the DC-based distribution network with the help of a multi-terminal (MT) structure and future mobility technologies such as electric ships. The purpose of this paper is to develop a platform for the EMS to efficiently operate the carbon-free island linked through DC networks, as shown in Figure 1.

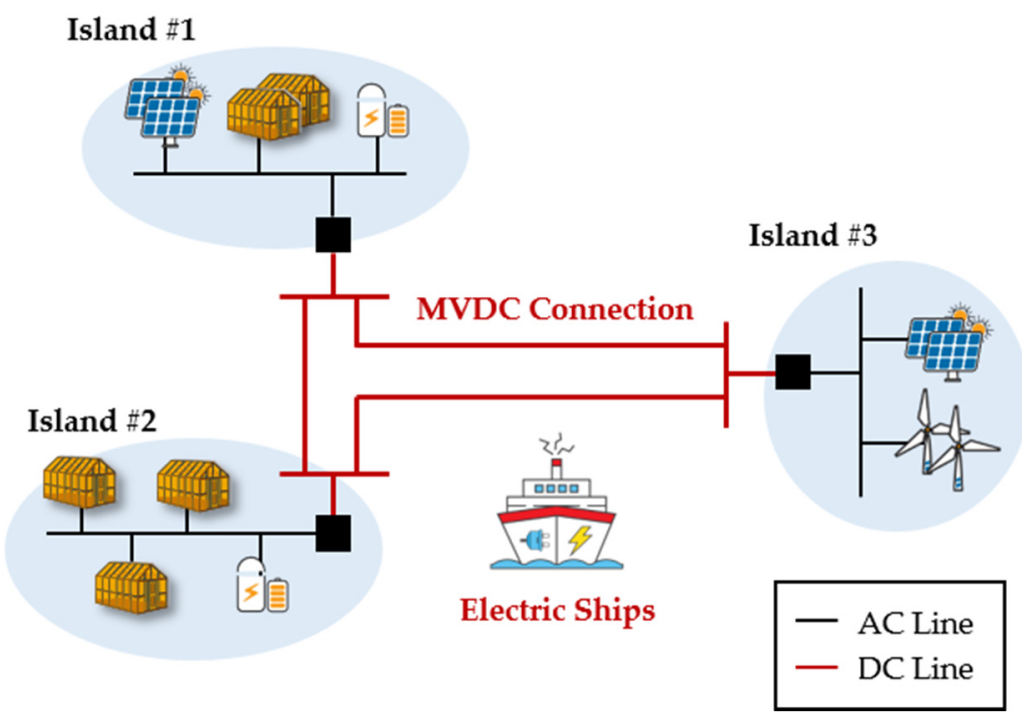

Figure 1. Diagram of the carbon-free island linked through DC networks.

\subsection{Configuration of the Operating System of the Carbon-Free Island Microgrid}

The carbon-free island MG is a new eco-friendly electric power supply system that connects multiple islands via DC-based distribution networks and electric ships, in order to improve the RE utilization rate of existing carbon-free islands and obtain economic feasibility. To operate the MG, a proper management system such as an EMS is required. Energy, voltage, and smart inverter/converter management functions are required in order to recognize and effectively operate the MG based on information on the state variables of the power network, such as voltage and current under normal state. Furthermore, protection coordination and fault location, isolation, and service restoration (FLISR) functions are required in order to respond to an emergency.

The main functions of the MG-EMS include power flow, voltage/current control, generation/load prediction, protection coordination, fault isolation, service restoration, energy management, and smart inverter/converter management. Power quality and voltage violations can be verified through power flow when conducting voltage and current control. The steady-state power supply stability can be secured by using energy 
management. In addition, by managing the smart inverter/converter, the carbon-free island MG can be operated systematically and efficiently, and reliability of the system's operation can be obtained even under abnormal conditions, via protection coordination and fault location, isolation, and service restoration (FLISR).

\section{Interoperability between Components in the MG-EMS}

\subsection{Configuration of the EMS Control Center}

EMSs are designed to efficiently operate a target system by acquiring and monitoring data measured in power facilities in real time. The EMS consists of a control center, communication devices, and field devices. The control center is composed of a frontend processor (FEP), communication protocol, middleware, database, human-machine interface (HMI), CIM, and applications. The FEP transmits information on the system under operation to the control center by communicating with terminal devices using a specific protocol through a wired or wireless communication network; it acquires data according to the scheduling cycle, responds to the unsolicited responses of field devices, and delivers control and measurement commands. DNP 3.0, commonly used in the power network field, is mainly utilized, and supports both polling and unsolicited responses.

Middleware delivers messages between components in the EMS, and supports requestreply and publish-subscribe message delivery methods. In the request-reply method, similar to the server-client method, the replier responds to requests from the requestor. The publish-subscribe method delivers messages via broadcast, and multiple subscribers receive a message when a publisher generates it. The DB systematizes, integrates, and manages data required for the EMS, and provides information related to system operations to the user through the user interface; it can also process control commands. Applications such as power flow (PF), service restoration (SR), topology processing (TR), state estimation (SE), etc., are utilized to efficiently operate the system. Figure 2 shows the components of the control center in an EMS.

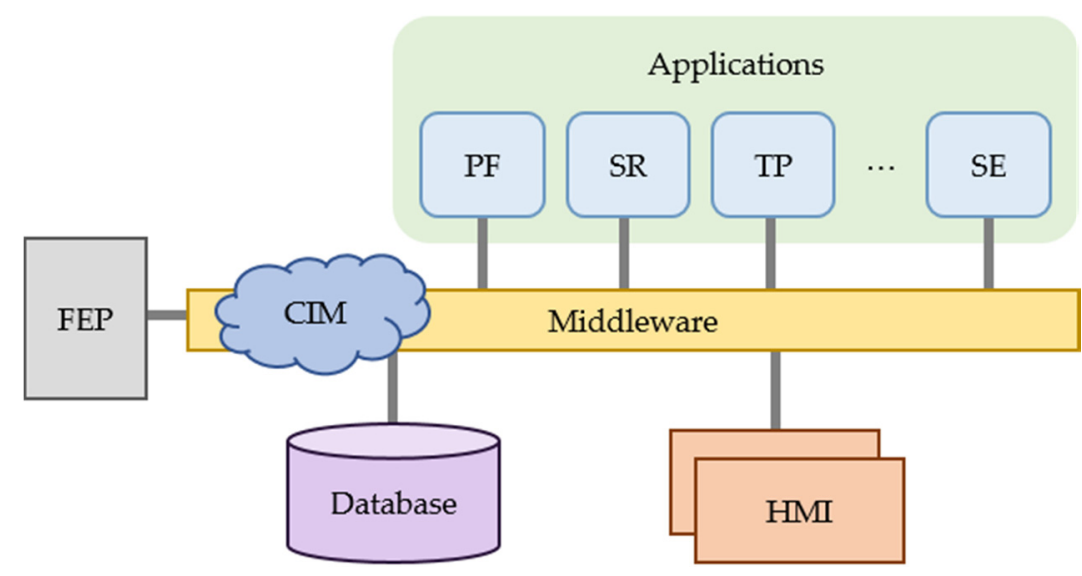

Figure 2. Components of the control center in an EMS.

\subsection{Purpose of the Interoperability}

Interoperability is ensured when information exchange is available between systems or components developed independently by different manufacturers without mutual consultation. Therefore, the subject who wants to exchange information should develop the module by referring to the standard without consulting with others, which means that interoperability relates to standards.

Since it is very difficult to predict changes in the industry, new systems should be developed to accommodate the changes without any trouble in exchanging data. Hence, the interface between systems should be implemented by using a standard. Generally, it is easy to implement a system and exchange information without using standards when the purpose of the system is clear and there is no possibility of changes in the system. 
However, it is necessary to use a standard for effective information exchange between several unspecified components. If the interface is implemented without using standards, consultation and changes in the interface should be made whenever a new component is added, thereby increasing the complexity of the system exponentially. On the other hand, if the interface is implemented based on standards, it is easy to expand the system in the future, although the initial development cost may be high and the development speed may be slow.

As indicated in Figure 3, the functions required for power network operations, such as service restoration (SR) applications, can be applied to other systems as well as the EMS for the carbon-free island MG. A system with interoperability has the advantage of being able to utilize the functions of other systems without any changes.

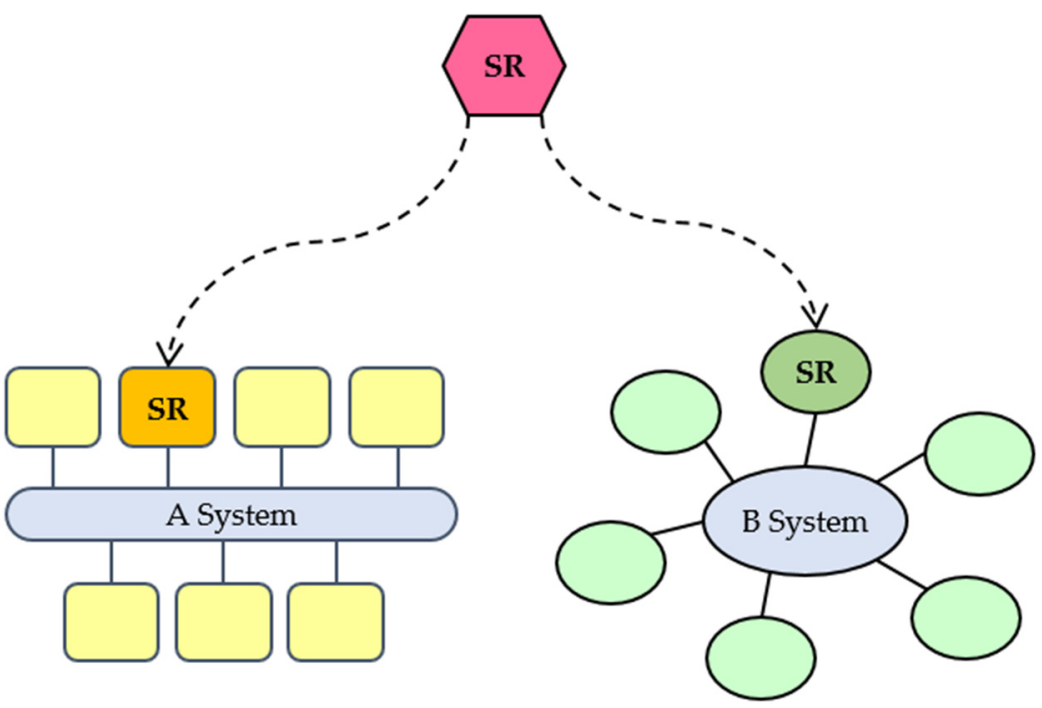

Figure 3. Concept of component reuse via interoperability.

\subsection{Subject of IEC TC57 Interoperability}

Interoperability can be applied to systems, components, and processes. The applied standards are different according to the characteristics of each level. The interoperability between systems aims for a loosely coupled system based on various programming languages, operating systems, protocols, and system management tools. Components in a loosely coupled system can be easily replaced with alternative components that provide the same services. Interoperability between components in the system, which are unit modules that can perform independent functions and be exchanged later, requires a standardized information exchange model between the components of the control center in a system such as a DMS, EMS, etc., and relatively high real-time response and reliability are also required. The issue of information exchange between intelligent electronic devices (IEDs) used in automation systems in the distribution field, or between IEDs and dataset devices (e.g., FEPs, data concentration units), is also applied to RE connections such as distributed generation, electric vehicles, ESSs, etc.

As shown in Figure 4, the IEC 61968, IEC 61970, and IEC 61850 standards are applied for the system, component, and process levels, respectively. In this paper, a method to secure interoperability between components in the carbon-free island MG-EMS is presented. IEC TC57 WG13 established IEC 61970, which defined the CIM-an abstract common information model for power system components. The interoperability between the application and other systems can be ensured if the application is developed by referring to the CIM, since it is presented in IEC 61970. 


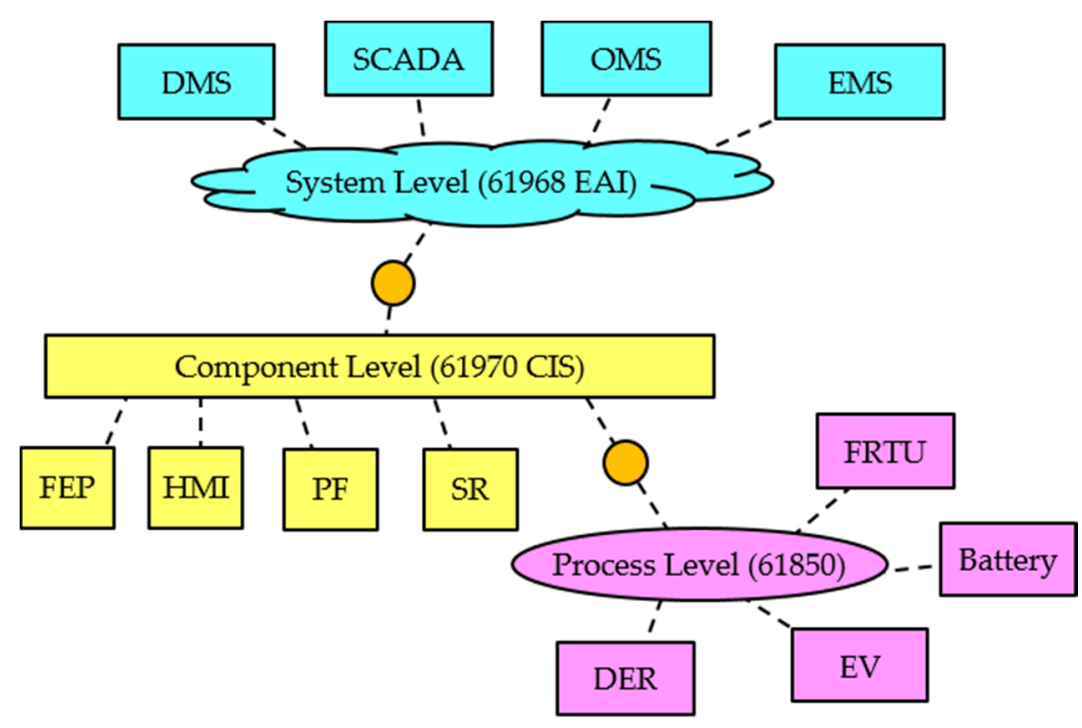

Figure 4. The applicable subjects of the interoperability.

\section{Proposed MG-EMS Platform for Securing Interoperability}

An information model for all data to operate a target system should be designed in order to ensure interoperability of the MG-EMS, and profile and payload-which are parts of the information model — should be created for each interface. Figure 5 shows the creation procedure of the information model, profile, and payload to ensure interoperability. As indicated in the figure, firstly, a complete CIM for interoperability is designed. A profile that can be created by the CIM enterprise architect (EA) is a part of the information model, and consists of class, attributes, and associations necessary for data input/output. Payload is developed by referring to the profile created, and it can be produced by processing the data input from the DB in the application into the CIM.

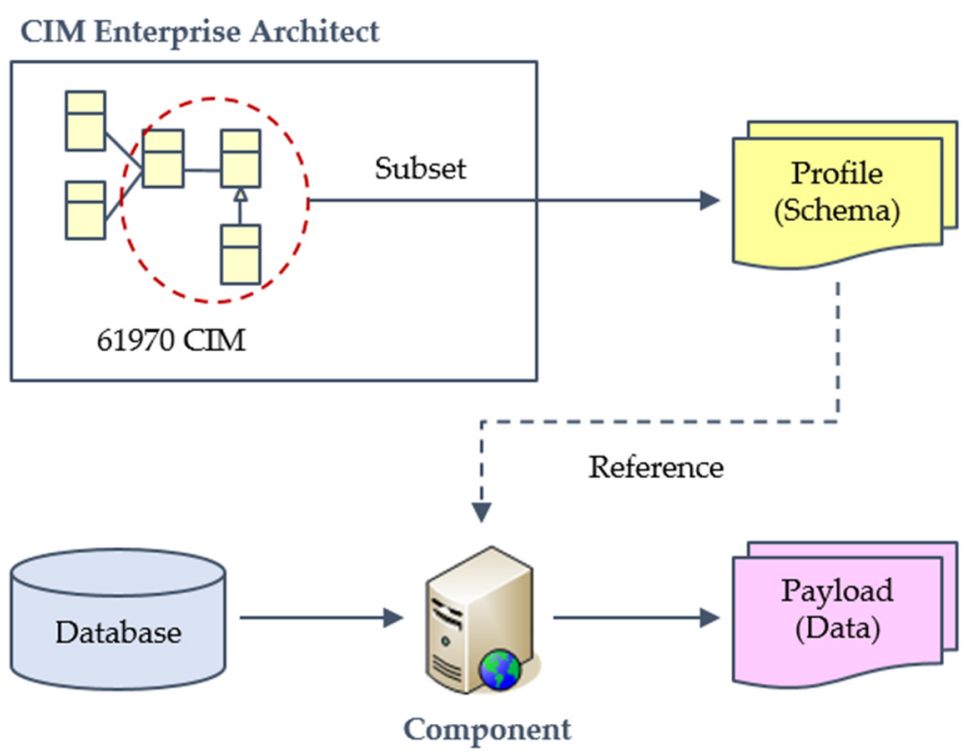

Figure 5. Data creation procedure based on the IEC 61970 CIM.

\subsection{Design of the Information Model Based on IEC 61970}

IEC 61970-301 provides the CIM, which is abstractly defined so that it can be commonly used for the components of a power system. Since the CIM is expressed as a unified modeling language (UML) class diagram, its contents can be easily shared with developers and system designers. UML is an object-oriented modeling technique, and class diagrams 
are expressed in the form of simple rectangles and arrows. Therefore, communication between system developers can be facilitated. By using the UML class diagram, the characteristics of each class and the relationships between the classes can be expressed. Class includes attributes, and the relationships between the classes are expressed by association, aggregation, etc. Interoperability between components can be secured by utilizing the information model, and is designed through use case definition, business object definition, gap analysis, and CIM extension procedures.

\subsubsection{Creation of Use Cases}

Table 1 indicates the interfaces for the functions required to operate the carbonfree island MG-EMS. To operate the system stably while supplying eco-friendly electric energy - such as PV and wind power-the functions presented in Table 1 should be mutually operated in an appropriately coordinated manner.

Table 1. List of the use cases for the carbon-free island MG.

\begin{tabular}{cc}
\hline No. & Use Case \\
\hline 1 & Data exchange for analyzing the state of the power network \\
2 & Data exchange for conducting voltage and power flow control \\
3 & Data exchange for prediction of generation and load \\
4 & Data exchange for energy demand management \\
5 & Data exchange for management of smart inverter/converter \\
6 & Data exchange for coordination between protective relays \\
7 & Data exchange for alarm processing and fault location \\
8 & Data exchange for establishment of service restoration plans \\
\hline
\end{tabular}

A diagram of the use case for data exchange to establish service restoration plans is presented in Figure 6. System topology, patterns of section load, distributed power generation, and ESS charging/discharging should be provided as requirements for establishing service restoration plans, and remote control of the switches installed in the power networks should be available. Participants consist of 'network operation', 'network model management', 'energy forecasting', and 'end device control'. To make sure that the resulting plan is acceptable, the amount of load in outage areas should be minimized, and critical loads such as hospitals should be the first to be restored. In addition, there should be no violations such as overvoltage, overload, etc., and the amount of switching for a given service restoration plan should be minimized.

Figure 7 shows the use case scenario for establishing service restoration plans. First, the network operation requests topology information and load/generation prediction data from the network model management, and establishes a service restoration plan considering the amount of load in outage areas, restoration of important loads, and the amount of switching during the service restoration. If there are any violations such as overvoltage or overload for the established plan, another service restoration plan is established instead; otherwise, the network operation delivers the established plan to the end device control.

\subsubsection{Design of the Business Object}

The business object-an abstracted object to implement the use case- of the use case for establishing service restoration plans defined in Section 4.1.1 is presented in Table 2. For this case, service restoration plans can be established by using an inner loop based on the load in outage areas, as well as by considering the charging/discharging capability of nearby ESSs and the state of the DC-based distribution network. For the service restoration application to operate in a system with interoperability secured, the business object indicated in Table 2 is required. By defining the business object, an information model can be designed, and it can be presented as a UML diagram consisting of classes, attributes, and associations. 
Use Case: Service Restoration

Precondition

- Load, DER, ESS charging/discharging pattern should be known

- Power system Topology should be known

- Switching remote control should be possible

\section{Purpose}

- Outage load minimization

- Important load priority restoration

- Switching number minimization

- No violation from a system (overvoltage, overcurrent, etc.)

\section{Participants}

- Network Model Management

- Network Operation

- Energy Forecasting

- End Device Control

\section{Key Performance Indicators}

- Outage load amount

- Important load outage amount

- Restoration plan switching number

- Violation number of system constraints

Figure 6. Diagram of the use case for establishing service restoration plans.

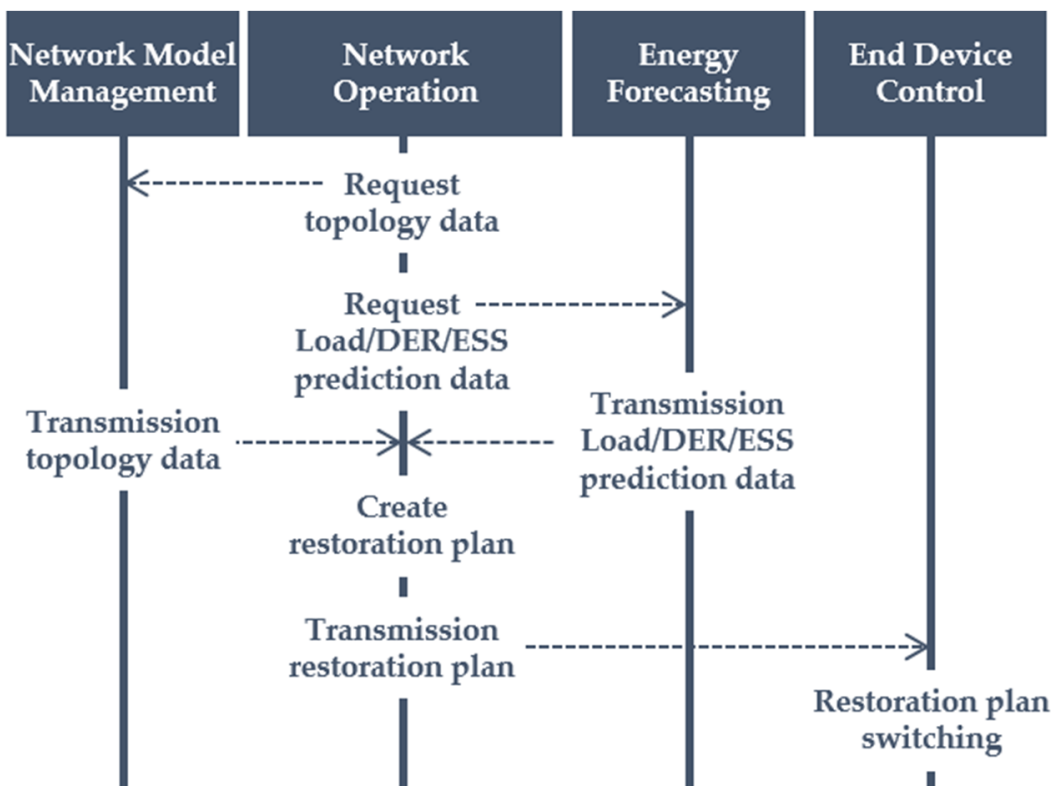

Figure 7. The scenario of the use case for establishing service restoration plans.

Table 2. The business object for the carbon-free island MG.

\begin{tabular}{cc}
\hline No. & Use Case \\
\hline 1 & Information on the topology of the power network \\
2 & Information on the outage area \\
3 & Information on the prediction of load patterns for each section \\
4 & Information on the prediction of ESS charging/discharging \\
5 & Information on the state of the DC-based power network \\
6 & Information on the amount of load for sections of the inner loop \\
7 & Information on the results of establishing service restoration plans \\
\hline
\end{tabular}




\subsubsection{Gap Analysis}

Information models that are not defined in IEC 61970 can be extended based on the results derived via gap analysis when creating business objects. Figure 8 shows an example of the CIM extension derived through the use case of service restoration. In the figure, the UML diagram of load pattern for conducting service restoration using an ESS is presented, along with the profile of the charging/discharging pattern of the ESS. The 'SectionLoad' class is an extended model to deliver data on section load, since the existing IEC 61970 CIM does not define it.

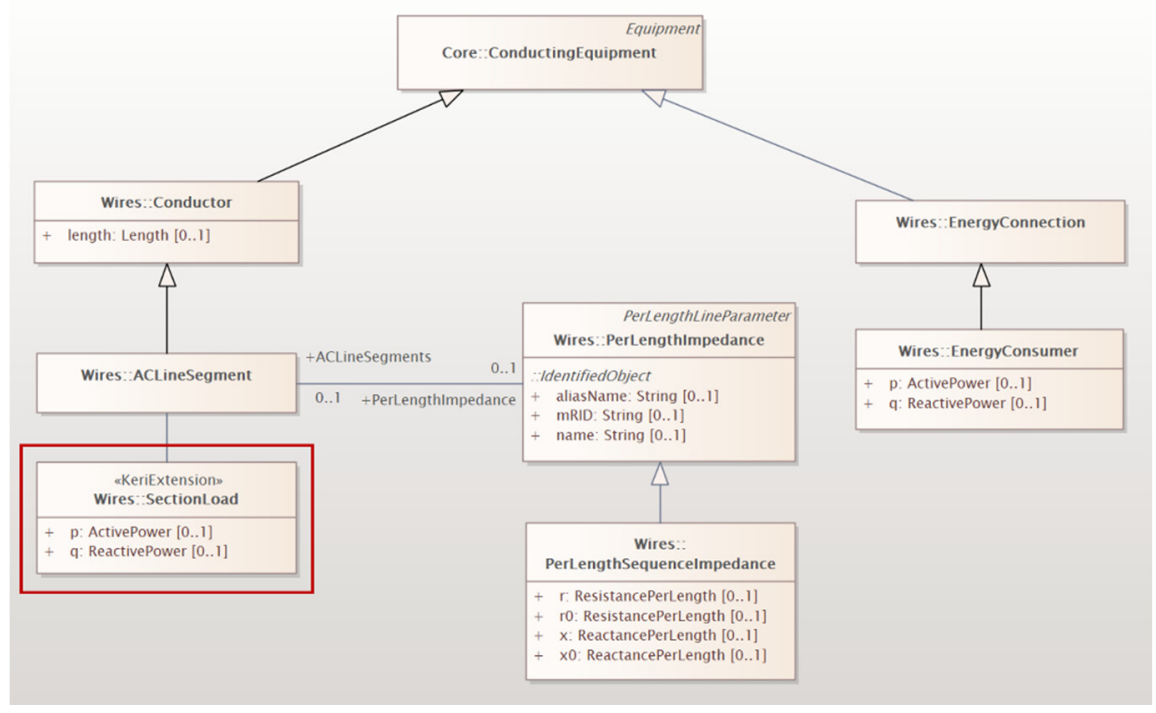

Figure 8. An example of gap analysis.

\subsection{Creation of CIM Profile/Payload}

Profiles for delivering data based on IEC 61970 can be created by selecting classes, attributes, and associations as a subset of the CIM. As shown in Figure 9, CIM EA-a software tool developed with the reference to IEC 61970-501 and World Wide Web Consortium (W3C) recommendations - can create profiles for interfaces to be input and output. Figure 9 shows the 'DCContrainment' profile extracted by utilizing the CIM EA tool. The profile represented by the UML diagram consists of classes, attributes, and associations. As shown in Figure 9, the profile of the 'DCContrainment' consists of classes such as 'ConnectivityNode', 'EquipmentContainer', 'Equipment', 'DCLine', 'DCConverterUnit', etc. The 'DCConverterUnit' includes the 'OperationMode' attribute, while the 'EquipmentContainer' aggregates the multiple 'Equipments'. In addition, the 'EquipmentContainer' generalizes the 'Substation', and the 'ConnectivityNodeContainer' and the 'ConnectivityNode' are under the association relationship.

The application creates a payload by converting the data in the DB into the CIM by referring to the profile created using CIM EA. The payload represents the data that are sent to the component for achieving the purpose of data transmission. The payload consists of a CIM structure, and contains data that are actually transmitted.

\subsection{Delivery of Data Based on the IEC 61970 CIM}

Results of CIM-based data modeling should be delivered in order to ensure the interoperability between components in the carbon-free island MG-EMS. Figure 10 shows the CIM-based data transfer process between two different components [4]. Data in CIM XML format to be delivered from 'A component' are exported by referring to the CIM $\mathrm{XML} /$ resource description framework (RDF) schema that defines the meaning and structure of data to be delivered as a profile. 'B component' imports the received payload-CIM XML format data—by referring to the CIM XML/RDF schema. 


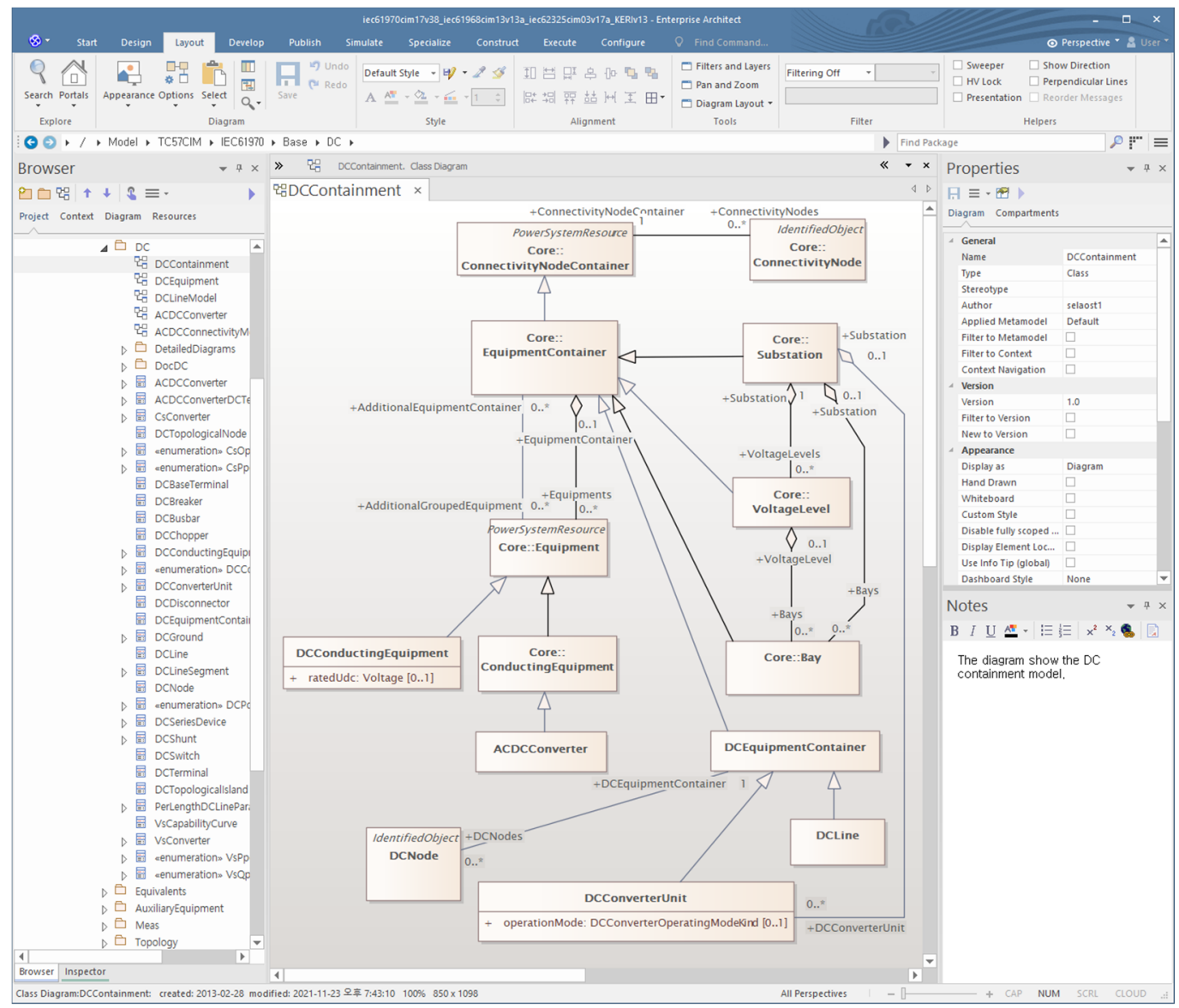

Figure 9. Creation of profiles using CIM EA.

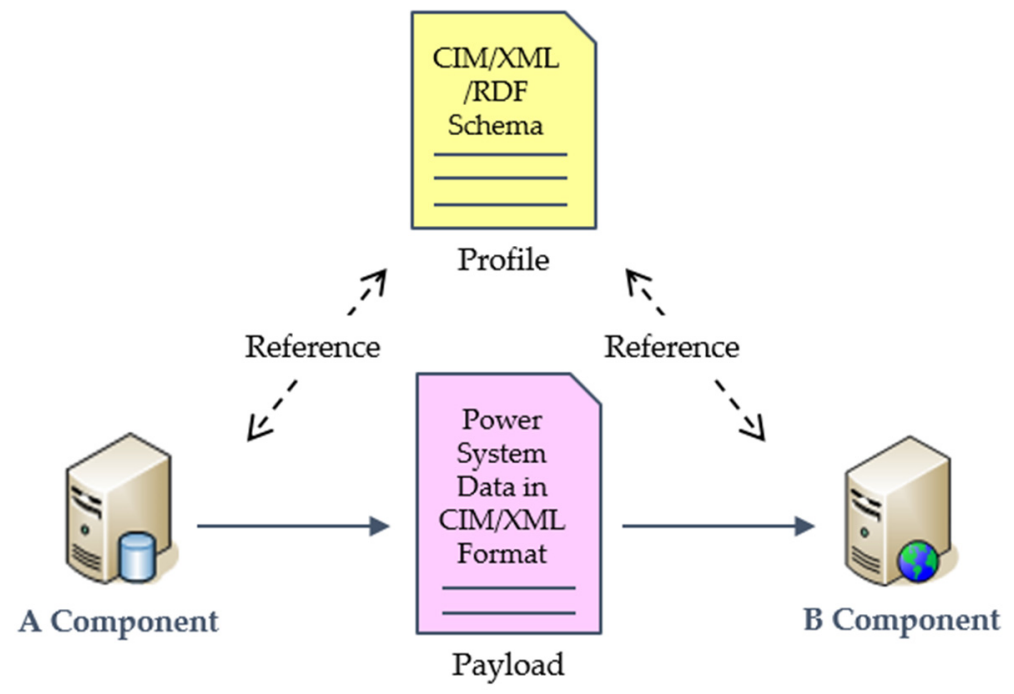

Figure 10. CIM-based data transfer process between two different components.

\section{Case Study}

A case study was performed to demonstrate the usefulness and flexibility of the development method of the carbon-free island MG-EMS for securing interoperability. The profile and payload for the MG-EMSs of carbon-free islands are created through the process presented in Section 4, dealing with the creation of the use case, the design of business objects, and the gap analysis. An MG-EMS prototype of the carbon-free island 
was developed to perform case studies for verifying the usefulness of the proposed method in ensuring interoperability. As shown in Figure 11, the MG-EMS prototype consists of an FEP emulator, middleware, a database, a user interface, and application programs. The FEP emulator supports the communication method based on the DNP 3.0 protocol, and the middleware developed based on ZeroMQ — which is an asynchronous messaging library, aimed at use in distributed or concurrent systems-supports the request-reply and publish-subscribe message delivery methods.

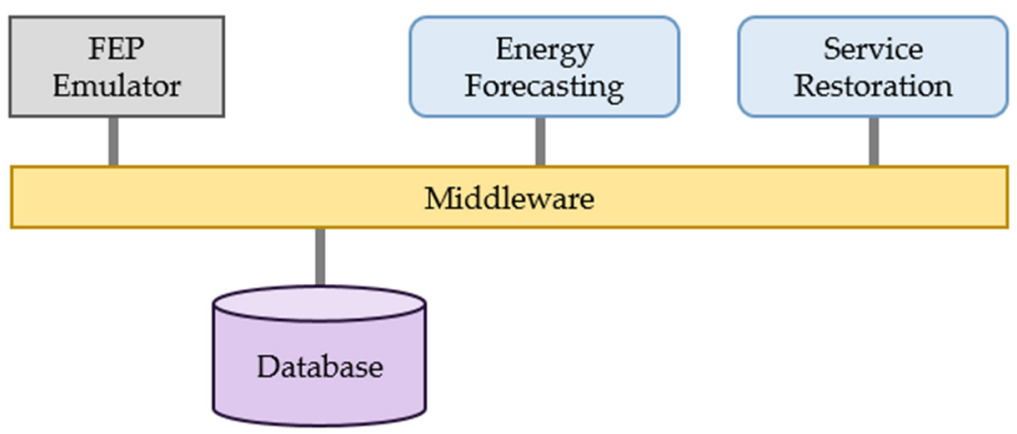

Figure 11. A prototype of the MG-EMS platform.

An example carbon-free island for the case study is presented in Figure 12. The system consists of 1 diesel generator, 1 ESS, 1 wind generator, 2 PV units, and 16 switches. The case study was conducted based on the scenarios for two use cases proposed in Table 1: the first scenario is the prediction of generation and load (No. 3), and the second scenario is the establishing of service restoration plans (No. 8). Figure 13 shows the derived final service restoration plan. As a result of performing the service restoration application, the fault section (A) surrounded by switches 2, 3, and 7 was separated, and the service restoration plan utilizing the ESS was derived to restore the service of the outage area (B) surrounded by switches $3,6,8$, and 9 [27].

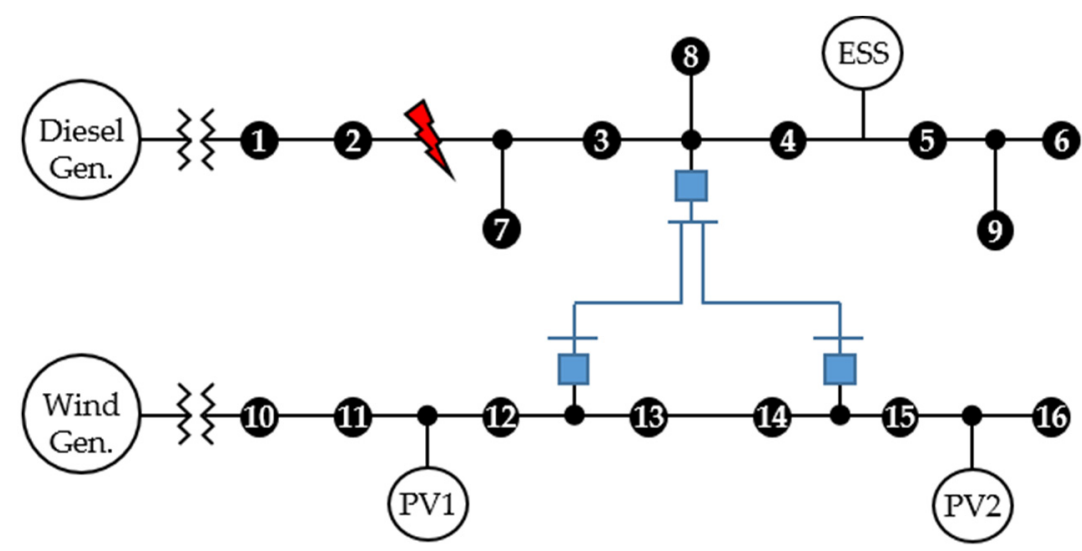

Figure 12. An example system for the case study.

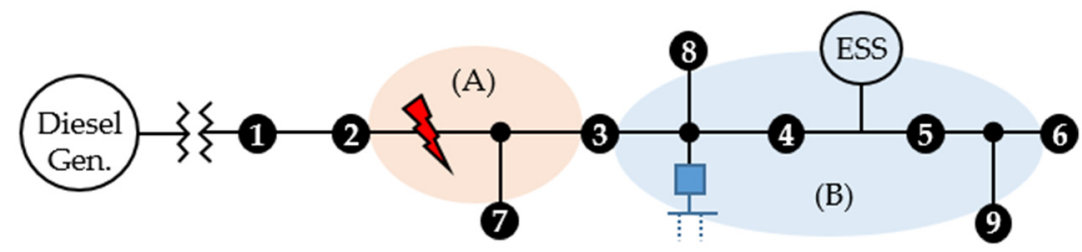

Figure 13. Service restoration plan using an ESS. 


\subsection{Scenario 1: Energy Forecasting}

Figure 14 shows the profile expressed in the UML diagram for the prediction of generation and load, which is extended by considering the requirements of the carbon-free islands. The profile- a subset of the entire CIM-is derived by using the CIM EA tool, as shown in Figures 14 and 15a. The profile for the prediction of generation and load consists of 'PowerElectronicsUnit', 'BatteryUnit', 'BasicIntervalSchedule', 'RegularIntervalSchedule', 'SeasonDayTypeSchedule', 'NonConformLoadSchedule', 'RegularTimePoint', and 'SectionLoad' classes. The 'SectionLoad' is a CIM extension class and, except for that, the rest are original CIM classes. The 'BasicIntervalSchedule' class has an association relationship with 'PowerElectronicsUnit' and 'SectionLoad'. Figures 14 and 15a represent the profile containing the metadata for the CIM, which explains its packages, classes, attributes, and associations. Figure 15b shows data exchanged between the components for the prediction of generation and load. The payload for the output from the energy forecasting application is created by referring to the CIM-based profile. The payload shown in Figure 15b, created by referring to the profile shown in Figure 15a, is transferred to the ZeroMQ-based middleware so that data can be exchanged with other components. The interoperability between the internal components of the MG-EMS is ensured by the middleware acting as a data transfer broker between the components.

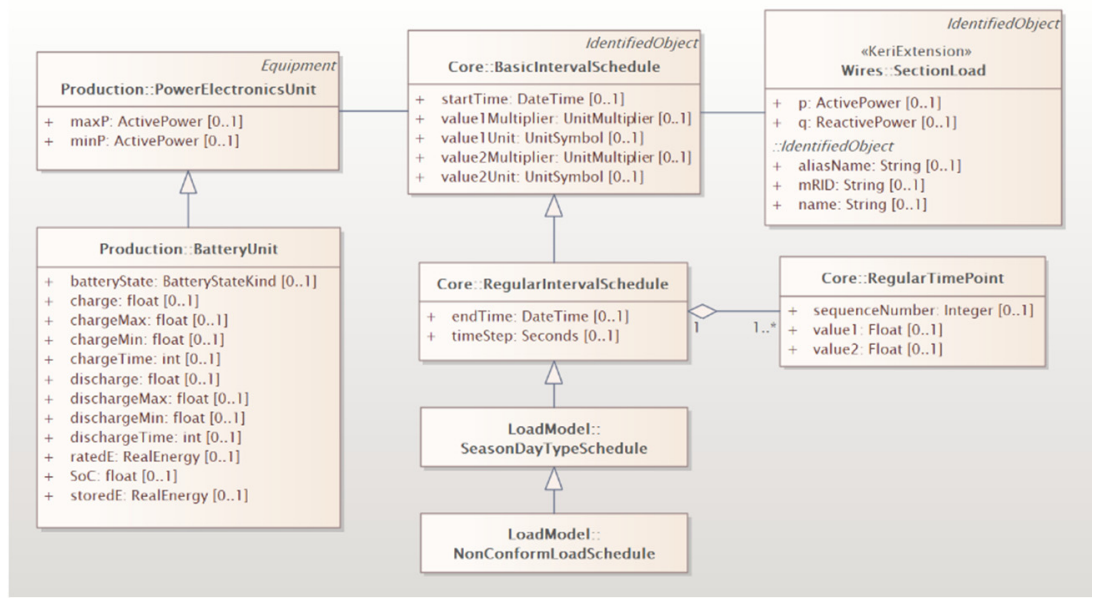

Figure 14. A UML diagram of the prediction of generation and load.

\subsection{Scenario 2: Service Restoration}

Figure 16 shows the profile expressed in the UML diagram for the service restoration plan using the ESS, which is extended by considering the requirements of the carbon-free islands. The profile is derived by using the CIM EA tool, as shown in Figures 16 and 17a. Figure $17 \mathrm{a}, \mathrm{b}$ show the profile and payload used for data exchange to operate the service restoration application, respectively. The profile for the service restoration plan using the ESS consists of 'Restoration', 'RestorationFuzzy', 'SectionGroup', 'Section', 'SectionLoad', 'Fuzzy', 'MembershipFunction', 'Fault', 'RestorationType', 'ACLineSegment', and 'BatteryUnit' classes. The 'ACLineSegment' and 'BatteryUnit' are original CIM classes, while the 'Restoration', 'RestorationFuzzy', 'SectionGroup', 'Section', 'SectionLoad', 'Fuzzy', 'MembershipFunction', 'Fault', and 'RestorationType' are CIM extension classes. The 'Restoration' class has an association relationship with 'Fault', 'RestorationFuzzy', and 'SectionGroup'. The 'SectionGroup' - meaning a unit section surrounded by automatic switches-is composed of multiple 'Section' and 'SectionLoad' classes. 


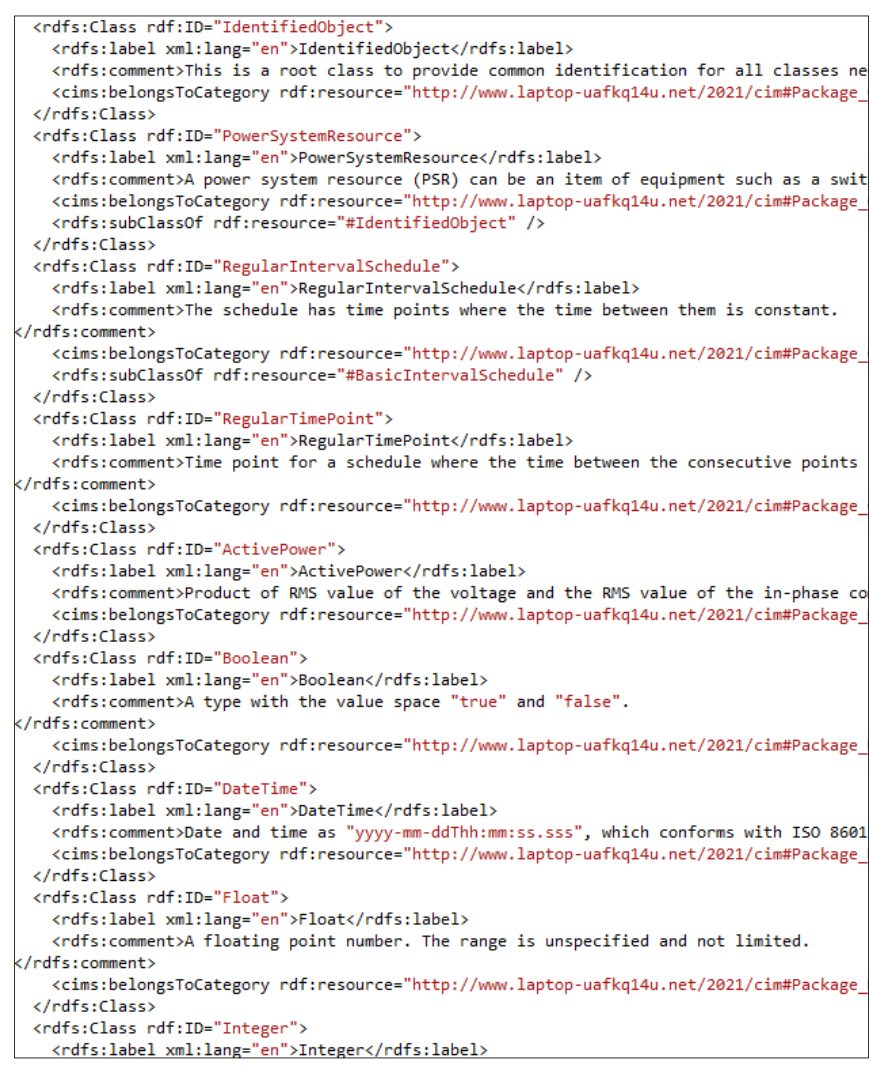

(a)

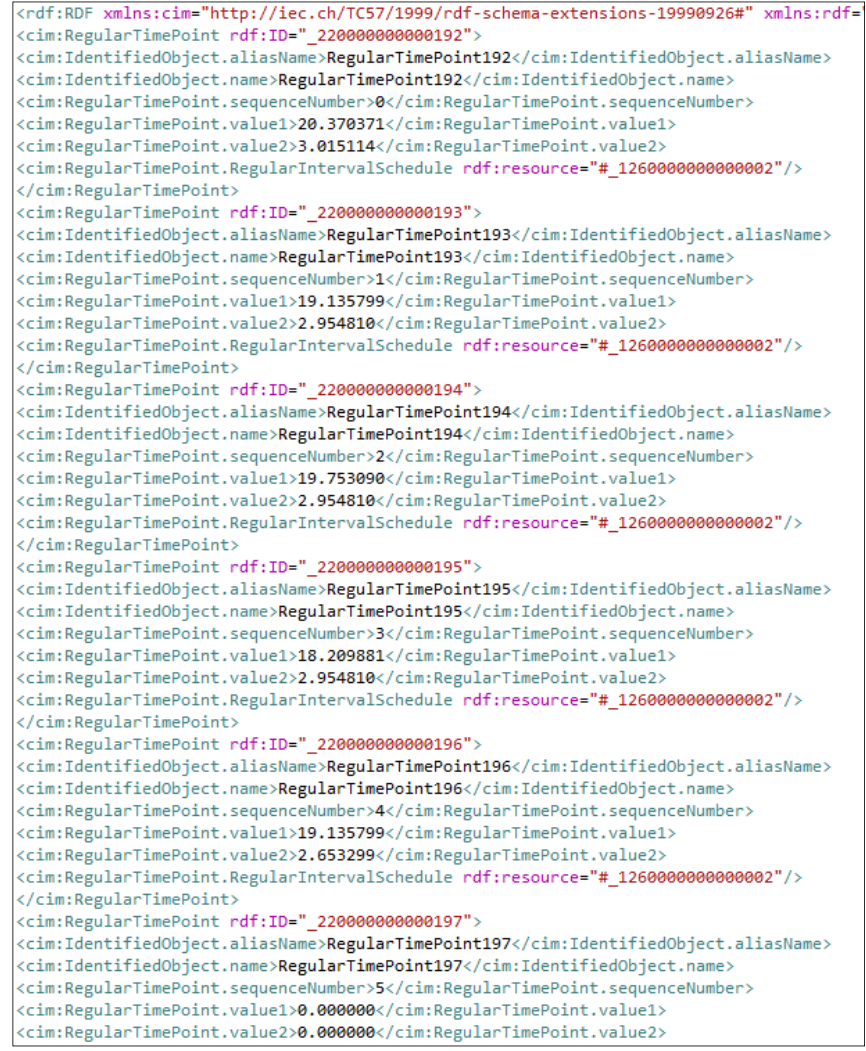

(b)

Figure 15. (a) Profile of the prediction of generation and load; (b) Payload of the prediction of generation and load.

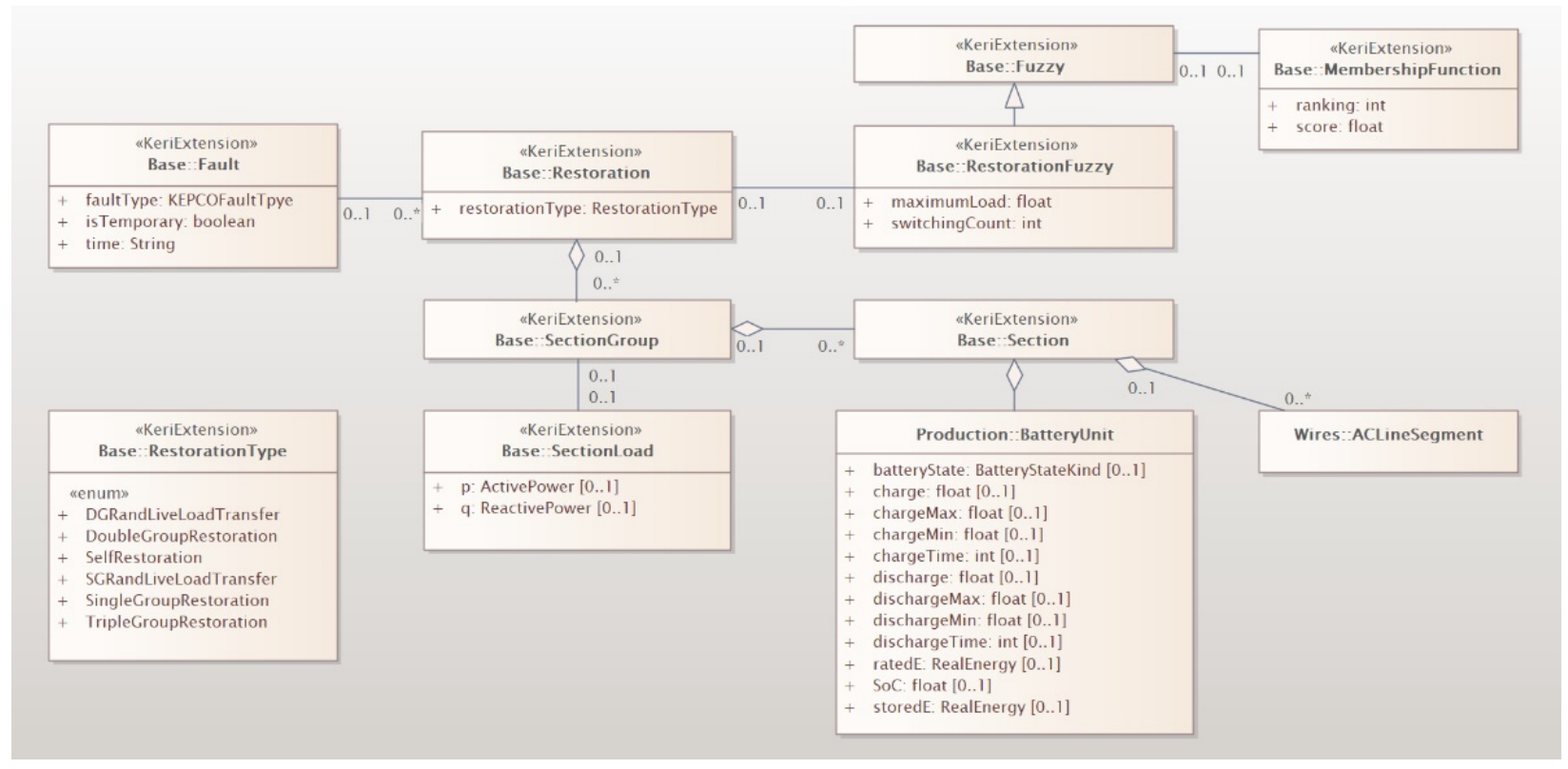

Figure 16. A UML diagram of the service restoration plan using the ESS. 


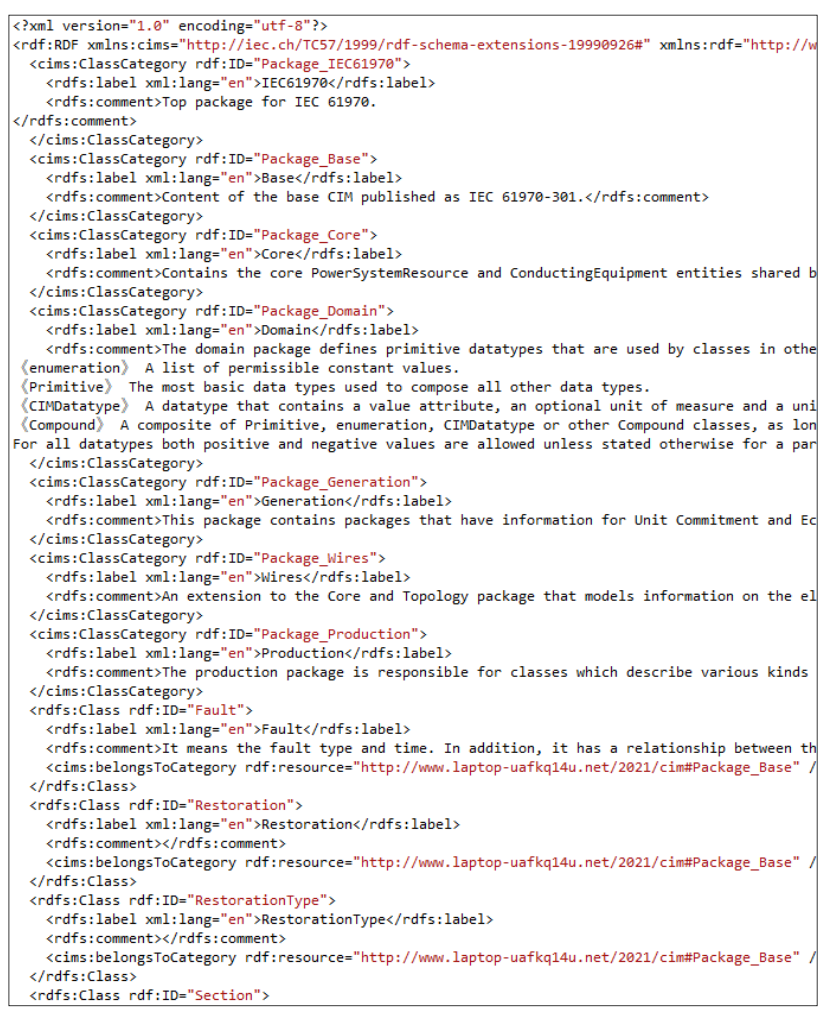

(a)

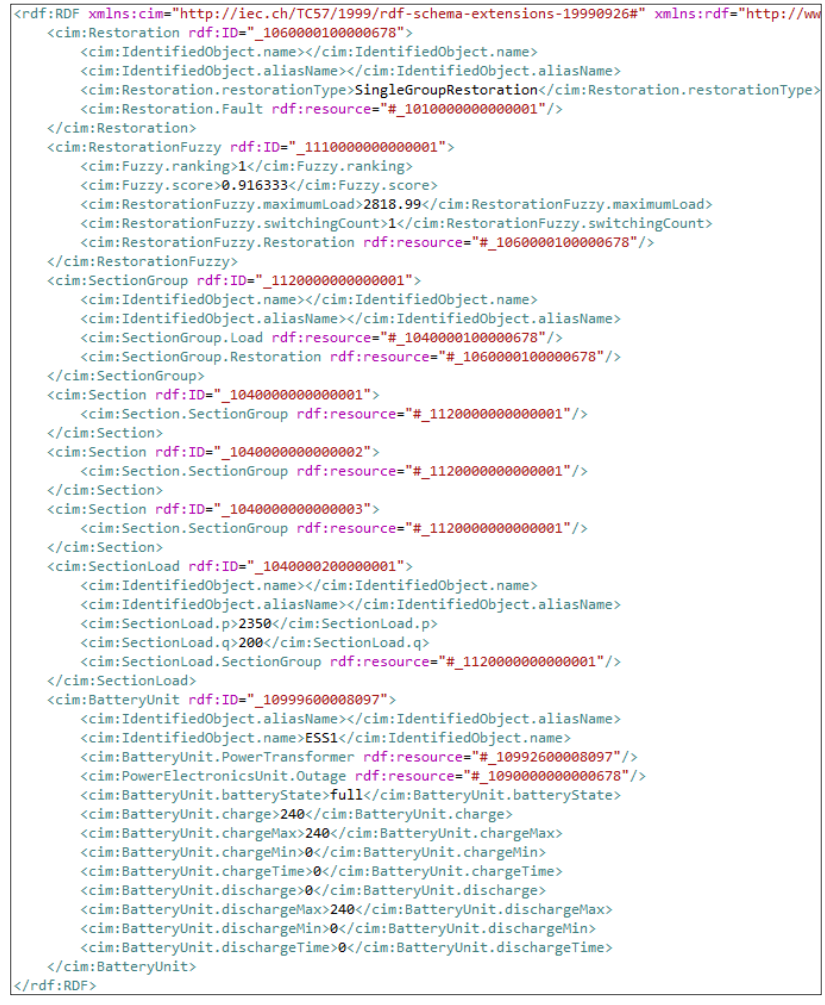

(b)

Figure 17. (a) Profile of the service restoration plan using the ESS; (b) Payload of the service restoration plan using the ESS.

Figure 18 shows the payload including information on the outage area, load pattern, ESS charging/discharging pattern, network topology, and service restoration plans. The load and ESS charging/discharging pattern in the form of payload, as shown in Figure 15b, are outputs of the energy forecasting application. The outage area, load pattern, ESS charging/discharging pattern, and network topology are received as inputs to the service restoration application. In addition, the service restoration application outputs the resulting service restoration plan in the form of payload, as shown in Figure 17b. All payloads that are inputs / outputs of the application are created by referring to the profile for each interface. Table 3 indicates the data size and data exchange speed for all standard interfaces required to operate the service restoration application. As shown in the table, the data size and data exchange speed are relatively reasonable given that EMSs operate in minutes.

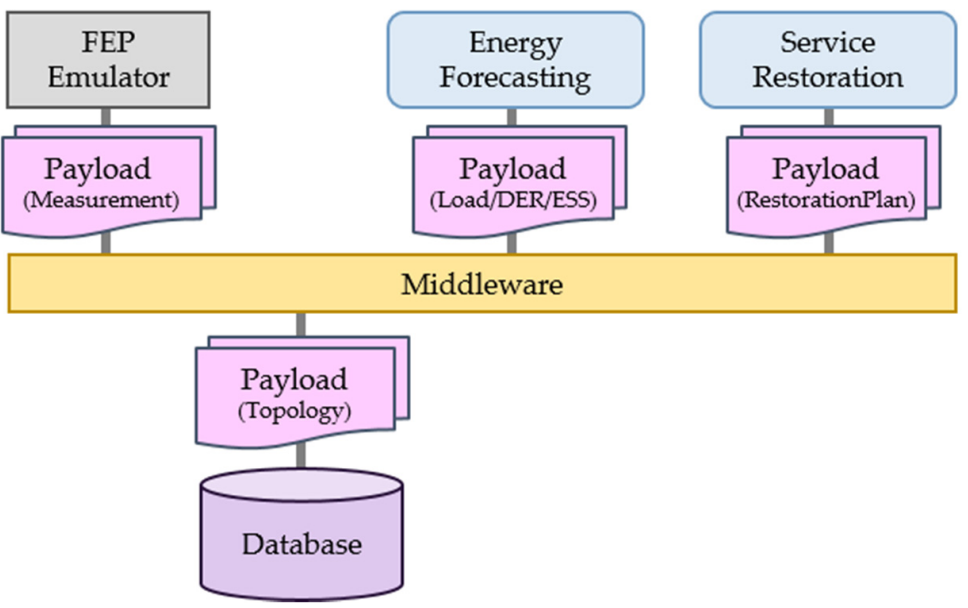

Figure 18. CIM-based data transfer through middleware. 
Table 3. Data size and speed by interface.

\begin{tabular}{cccccc}
\hline No. & Sender & Receiver & Payload & Size (kB) & Speed (s) \\
\hline 1 & Database & ServiceRestoration & Topology & 45.7 & 1.9 \\
2 & FEPEmulator & ServiceRestoration & Measurement & 44.2 & 1.2 \\
3 & EnergyForecasting & ServiceRestoration & Load/DER/ESS & 50.9 & 4.2 \\
4 & ServiceRestoration & UserInterface & RestorationPlan & 18.6 & 1.5 \\
\hline
\end{tabular}

The interoperable platform proposed in this paper can be applied not only to the energy forecasting and service restoration applications, but also to other applications, by utilizing the standard interface. For any interconnection between components, the same approach presented in this paper-which includes use case analysis, design of business objects, gap analysis, and CIM extension - can be utilized for securing interoperability. Through the case study, it was confirmed that the MG-EMS operates smoothly even if the CIM-based interoperability method is applied. Since the MG-EMS proposed in this paper developed the standard interface, it is expected that applications implemented based on the standard interface will be able to be accommodated in the future.

\section{Conclusions}

With the expansion of RE and new energy industries, there is a need for securing the interoperability of carbon-free island MG-EMSs. A method for ensuring interoperability between components in the MG-EMS is proposed based on IEC 61970 and 61968 CIM. We analyzed the previously defined CIM and created use cases according to the functions of a target system. In addition, detailed diagrams and scenarios for the use cases were created, and the business objects for executing use cases were designed. Through case studies, the feasibility of the proposed method was verified by applying the method to secure interoperability for service restoration and load/generation prediction use cases. Even if IEC61970 CIM-based standard interfaces were applied, it was verified that the load and generation prediction results and the service restoration plan were created and delivered within $4.2 \mathrm{~s}$ and $1.5 \mathrm{~s}$, respectively, and the components in the MG-EMS operated smoothly. It is expected that it will be possible to build a platform that responds flexibly to future changes by securing interoperability between components in the carbon-free island MG-EMS.

Author Contributions: Conceptualization, J.H. and Y.-S.O.; methodology, J.H.; software, J.-G.A.; validation, J.-U.S. and J.H.; formal analysis, Y.-S.O.; investigation, J.-U.S.; resources, J.-U.S.; data curation, J.H.; writing—original draft preparation, J.H.; writing—review and editing, Y.-S.O.; visualization, J.-G.A.; supervision, J.-H.J.; project administration, J.-H.J.; funding acquisition, J.-H.J. All authors have read and agreed to the published version of the manuscript.

Funding: This research was supported by Korea Electrotechnology Research Institute (KERI) Primary research program through the National Research Council of Science \& Technology (NST) funded by the Ministry of Science and ICT (MSIT) (No. 21A01027).

Conflicts of Interest: The authors declare no conflict of interest.

\section{References}

1. Government of South Korea. 2050 Net Zero Promotion Strategy; Government of South Korea: Seoul, Korea, 2020.

2. Ministry of Trade, Industry and Energy. The 5th Basic Plan for the Development, Use, and Distribution of New and Renewable Energy Technologies; Ministry of Trade, Industry and Energy: Seoul, Korea, 2020.

3. Ministry of Trade, Industry and Energy. The Era of the 4th Industrial Revolution, Policy Tasks for the Creation of New Industries; Ministry of Trade, Industry and Energy: Seoul, Korea, 2016.

4. IEC. IEC 61970-301, Energy Management System Application Program Interface (EMS-API)—Part 301: Common Information Model (CIM) Base; IEC: Geneva, Switzerland, 2020.

5. Santodomingo, R.; Rodriguez-Mondejar, J.A.; Sanz-Bobi, M.A. Using Semantic Web Resources to Translate Existing Files Between CIM and IEC 61850. IEEE Trans. Power Syst. 2012, 27, 2047-2054. [CrossRef] 
6. $\quad$ Lee, B.; Kim, D.-K.; Yang, H.-S.; Jang, H.; Hong, D.; Falk, H. Unifying Data Types of IEC 61850 and CIM. IEEE Trans. Power Syst. 2014, 30, 448-456. [CrossRef]

7. Wu, J.; Cheng, Y.; Schulz, N.N.; Ginn, H.L. The impact of standardized models, programming interfaces, and protocols on a shipboard power system. IEEE Trans. Ind. Appl. 2008, 44, 455-462. [CrossRef]

8. Ravikumar, G.; Yemula, P.K.; Khaparde, S.A. Graphics model for power systems using layouts and relative coordinates in CIM framework. In Proceedings of the 2014 IEEE PES General Meeting Conference \& Exposition, National Harbor, MD, USA, 27-31 July 2014; pp. 1932-5517.

9. Hargreaves, N.B.; Pantea, S.M.; Carter, A.; Taylor, G.A. Foundations of a Metamodel Repository for Use with the IEC Common Information Model. IEEE Trans. Power Syst. 2013, 28, 4752-4759. [CrossRef]

10. Santodomingo, R.; Rohjans, S.; Uslar, M.; Rodriguez-Mondejar, J.A.; Sanz-Bobi, M.A. Facilitating the Automatic Mapping of IEC 61850 Signals and CIM Measurements. IEEE Trans. Power Syst. 2013, 28, 4348-4355. [CrossRef]

11. Ten, C.-W.; Wuergler, E.; Diehl, H.-J.; Gooi, H.B. Extraction of Geospatial Topology and Graphics for Distribution Automation Framework. IEEE Trans. Power Syst. 2008, 23, 1776-1782. [CrossRef]

12. Gómez, F.J.; Vanfretti, L.; Olsen, S.H. CIM-compliant power system dynamic model-to-model transformation and modelica simulation. IEEE Trans. Ind. Inform. 2018, 14, 3989-3996. [CrossRef]

13. Britton, J.P.; deVos, A.N. CIM-based standards and CIM evolution. IEEE Trans. Power Syst. 2005, 20, 758-764. [CrossRef]

14. Pradeep, Y.; Seshuraju, P.; Khaparde, S.A.; Joshi, R.K. CIM-Based Connectivity Model for Bus-Branch Topology Extraction and Exchange. IEEE Trans. Smart Grid 2011, 2, 244-253. [CrossRef]

15. Wang, X.; Schulz, N.; Neumann, S. CIM extensions to electrical distribution and CIM XML for the IEEE radial test feeders. IEEE Trans. Power Syst. 2003, 18, 1021-1028. [CrossRef]

16. Chandramohan, L.S.; Ravikumar, G.; Doolla, S.; Khaparde, S.A. Business Process Model for Deriving CIM Profile: A Case Study for Indian Utility. IEEE Trans. Power Syst. 2014, 30, 132-141. [CrossRef]

17. Georg, H.; Muller, S.C.; Rehtanz, C.; Wietfeld, C. Analyzing Cyber-Physical Energy Systems: The INSPIRE Cosimulation of Power and ICT Systems Using HLA. IEEE Trans. Ind. Inform. 2014, 10, 2364-2373. [CrossRef]

18. Zhu, J.; Zhuang, E.; Ivanov, C.; Yao, Z. A data-driven approach to interactive visualization of power systems. In Proceedings of the 2012 IEEE Power and Energy Society General Meeting, San Diego, CA, USA, 22-26 July 2012.

19. Ravikumar, G.; Khaparde, S.A. A Common Information Model Oriented Graph Database Framework for Power Systems. IEEE Trans. Power Syst. 2016, 32, 2560-2569. [CrossRef]

20. McMorran, A.; Ault, G.; Morgan, C.; Elders, I.; McDonald, J. A common information model (CIM) toolkit framework implemented in java. IEEE Trans. Power Syst. 2006, 21, 194-201. [CrossRef]

21. Kim, D.; Yang, H.; Jang, H.; Hong, D.; Falk, H.; Kim, S.; Lee, B. A metamodeling approach to unifying IEC 61850 and IEC 61970. In Proceedings of the 2013 IEEE PES Innovative Smart Grid Technologies Conference (ISGT), Washington, DC, USA, 24-27 February 2013; pp. 1-6.

22. Cao, J.; Peng, M.; Guo, C.; Sun, W.; Xin, J. CIM-based information model for wide area measurement system. In Proceedings of the 2009 International Conference on Sustainable Power Generation and Supply, Nanjing, China, 6-7 April 2009; pp. 1-5.

23. Liu, L.; Yu, H.; Xu, X. Distribution circuit information model and its application. In Proceedings of the 2012 Second International Conference on Intelligent System Design and Engineering Application, Sanya, China, 6-7 January 2012; pp. $1243-1246$.

24. Wang, C.; Chen, Q.; Bo, R.; Huang, X.; Hu, M.; Yang, L. A new CIM-based cross-platform graphic system for power system applications. In Proceedings of the 2008 Third International Conference on Electric Utility Deregulation and Restructuring and Power Technologies, Nanjing, China, 6-9 April 2008; pp. 846-851.

25. Bennaceur, A.; Issarny, V. Automated Synthesis of Mediators to Support Component Interoperability. IEEE Trans. Softw. Eng. 2014, 41, 221-240. [CrossRef]

26. Georg, H.; Dorsch, N.; Putzke, M.; Wietfeld, C. Performance evaluation of time-critical communication networks for smart grids based on IEC 61850. In Proceedings of the 2013 IEEE INFOCOM, Turin, Italy, 14-19 April 2013; pp. 3417-3422.

27. Su, W.; Wang, J. Energy Management Systems in Microgrid Operations. Electr. J. 2012, 25, 45-60. [CrossRef]

28. Shen, J.; Jiang, C.; Liu, Y.; Wang, X. A Microgrid Energy Management System and Risk Management under an Electricity Market Environment. IEEE Access 2016, 4, 2349-2356. [CrossRef]

29. Palma-Behnke, R.; Benavides, C.; Lanas, F.; Severino, B.; Reyes-Chamorro, L.; Llanos, J.; Saez, D. A Microgrid Energy Management System Based on the Rolling Horizon Strategy. IEEE Trans. Smart Grid 2013, 4, 996-1006. [CrossRef]

30. Popovic, D.S.; Varga, E.; Perlic, Z. Extension of the Common Information Model with a Catalog of Topologies. IEEE Trans. Power Syst. 2007, 22, 770-777. [CrossRef]

31. Moseley, J.D.; Mago, N.V.; Sarma, N.D.R.; Grady, W.M.; Santoso, S. Extending CIM Standards to Support Exchange of Ratings on Dynamically Rated Equipment. IEEE Trans. Power Syst. 2015, 31, 296-303. [CrossRef] 